\title{
Producción audiovisual universitaria: espacios de innovación docente en Iberoamérica
}

\section{(Audiovisual Production in University: Spaces for Teaching Innovation in Iberoamerica)}

\author{
María Toscano-Alonso \\ Ignacio Aguaded Gómez \\ Universidad de Huelva (España) \\ Edna M. Manotas Salcedo \\ Universidad del Norte (Colombia) \\ Silvia C. Farias-Gaytán \\ Tecnológico de Monterrey (México)
}

DOI: $\underline{\text { https://doi.org/10.5944/ried.25.1.30611 }}$

\section{Cómo referenciar este artículo:}

Toscano-Alonso, M., Aguaded Gómez, J. I., Manotas Salcedo, E. M., y FariasGaytán, S. C. (2022). Producción audiovisual universitaria: espacios de innovación docente en Iberoamérica. RIED. Revista Iberoamericana de Educación a Distancia, 25(1), pp. 41-58. https://doi.org/10.5944/ried.25.1.30611

\section{Resumen}

La incorporación de los nuevos medios en el ámbito educativo aparece cada vez con más frecuencia. La docencia universitaria se vale cada vez más de estos materiales audiovisuales, digitales y multimedia para proporcionar una mejor experiencia en el aula. Para la producción de estos materiales se han creado espacios destinados a su desarrollo, no obstante, existe falta de consenso en el nombre atribuido a los mismos. En este estudio se ha realizado, en primer lugar, una revisión bibliográfica relativa a la producción de contenidos audiovisuales educativos y a los espacios en los que estos se desarrollan para contextualizar de manera previa a las entrevistas en profundidad. Con el objetivo de conocer y ahondar en las propuestas audiovisuales que se están dando en el ámbito iberoamericano, se ha entrevistado a personas responsables de estos espacios de innovación en tres universidades. Con lo que se ha podido concluir que las tres instituciones dedican recursos humanos, equipamiento y espacio especializado para el desarrollo de vídeo. El acompañamiento pedagógico es necesario para delinear la intención educativa de los materiales a desarrollar y es necesario empoderar tanto a docentes como alumnos en el desarrollo y curaduría de materiales. Los resultados pueden ser de utilidad para otras universidades y centros interesados en incursionar en la producción 
de vídeo, así como para oportunidades de colaboración que coadyuven a enriquecer la experiencia de aprendizaje y la formación docente.

Palabras clave: tecnologías educativas; edu-comunicación; e-learning; medialab; producción audiovisual.

\begin{abstract}
The inclusion of new media in the field of education is appearing more and more frequently. University teaching is increasingly utilizing these audiovisual, digital and multimedia resources to provide a better learning experience. For the production of these materials, designated spaces have been created, however, there is a lack of consensus regarding the name attributed to them. In this study, we carried out a bibliographical review of the production of educational audiovisual content and the spaces devoted to this end in order to contextualise it prior to the in-depth interviews. With the aim of getting to know and delve deeper into the audiovisual proposals that are taking place in the Ibero-American sphere, we interviewed the people responsible for these innovative spaces in three universities. We concluded that the three institutions dedicated human resources, equipment and specialised spaces for video development; pedagogical support is necessary to outline the educational intention of the materials to be created; and it is necessary to empower both teachers and students in the development and curation of materials. The results may be useful for other universities and centres interested in venturing into video production, as well as for collaboration opportunities to enrich the learning experience and teacher training.
\end{abstract}

Keywords: educational technologies; educommunication; e-learning; medialab; audiovisual production.

Los centros de producción de materiales educativos digitales tienen distintas denominaciones dependiendo de la institución que lo promueva. Algunos son catalogados como laboratorios de innovación digital -medialabs, edulabs, videolabs-, otros como fábricas de contenidos o unidades de apoyo dentro de centros de formación docente. Los contenidos, a su vez, han ido evolucionando de acuerdo con la tecnología, las plataformas y los modelos pedagógicos en tendencia. Esto depende, en gran medida, del perfil de cada institución, su enfoque y el presupuesto con el que se cuente (Guerrero-Romera et al., 2021; Hajhashemi et al., 2018).

De acuerdo con esto, es evidente la evolución conceptual que ha experimentado el vídeo educativo, no solo con el advenimiento de nuevas tecnologías, sino con las transformaciones en los enfoques pedagógicos (Aparici, 1997; García, 2014). En este sentido, se habla de una primera etapa -década de los 60- en la cual el enfoque fue más funcionalista (Fedorov, 2010). Este planteamiento estaba más centrado en el uso de películas en cineclubes para el análisis de la estética, la semiótica de la imagen, los lenguajes expresivos y para enseñar teorías de la comunicación. 
Luego se dieron iniciativas variadas en Europa en relación con el uso de medios de comunicación, especialmente como herramientas para desarrollar un pensamiento crítico frente a los mensajes televisivos. A esta corriente se le denominó 'Media Literacy' (Buckingham, 2003; Gabelas, 2007). Esta perspectiva tuvo su eje central en la educación presencial (Bravo-Ramos, 1996; Cebrián, 2002; Daza, 2012; Ferrés, 1992).

En ese momento, surgieron distintas clasificaciones para los vídeos educativos, como vídeo-lección, vídeo motivador, vídeo de apoyo, documentales, narrativos, lección monoconceptual, lección temática y vídeos motivadores (Cebrián, 2002). Al paso de estas evoluciones, a mediados de la década de los 80, la educación a distancia (Anderson y Dron, 2011) va cambiando también de formatos, de impresos y radio, pasando a las plataformas Learning Management System y LMS, donde el vídeo se convierte en una pieza esencial de los cursos en línea (Accino y Cebrian, 2008). Sin embargo, algunos autores afirman que esto no representó en sí mismo una evolución. Para Laaser y Toloza (2017), el uso continuo de los medios audiovisuales se está quedando atrás en términos de calidad. Esta preocupación va de la mano con la transformación de los enfoques pedagógicos, pasando de escenarios de aprendizaje unidireccionales al constructivismo, aprendizaje colaborativo, conectivismo, realidad virtual, entre otros (Scavarelli et al., 2021); es decir, el eje del proceso de enseñanzaaprendizaje no es el contenido, sino el cómo se aprende. Con esta perspectiva han ido evolucionando las plataformas, sin embargo, gran parte de los vídeos educativos se centran en historias tipo estándar: busto parlante "talking head". En este orden de ideas, distintos autores, plantean la existencia de muchos retos para lograr que el vídeo educativo sea usado en un proceso de mediación en el aprendizaje y no como un mero canal para transmitir contenidos.

En consecuencia, el diseño y producción de un vídeo requiere un objetivo de aprendizaje claro, así como contenidos y elementos audiovisuales definidos (Salinas-Ibáñez y De-Benito, 2020). Algunos estudios de neurociencia sugieren que el uso de recursos receptivos como el vídeo o la televisión, en los cuales el usuario principalmente recibe y comprende el contenido presentado, son un mejor medio para presentar conceptos que implican identificar causalidad y contexto. En cambio, si el objetivo de aprendizaje es ayudar a recordar asociaciones específicas, una aplicación interactiva es la más adecuada (Anderson y Davison, 2019; Hung et al., 2018). También se destaca que la presentación visual de contenidos procedimentales es más efectiva a través del formato audiovisual comparada con el formato escrito (texto) (Carmichael et al., 2018). Por lo anterior, el concepto de vídeo debe adaptarse, con el fin de cubrir las necesidades de aprendizaje planteadas.

Asimismo, cabe anotar que el vídeo educativo pasó de ser estático a convertirse en una serie de relaciones entre fragmentos con los formatos transmedia, inclusive, usando redes sociales, entre otros aplicativos, y en donde la interacción es la protagonista, aspecto clave para enfoques pedagógicos constructivistas (Cebrián, 2009; Edu Trends, 2017; Hovious et al., 2021; Koumi, 2016). De igual manera, la 
tecnología móvil y portátil ha permitido que los docentes produzcan sus propios vídeos demanerafácil con aplicaciones gratuitas, y a su vez, usan YouTubey Bancos deObjetos Virtuales de Aprendizaje para compartir material con otros profesores alrededor del mundo. En muchos casos ya no dependen de grandes equipos de producción para lograr sus materiales. El cambio en los lenguajes expresivos demuestra avances en la forma de narrar y comunicarse para la docencia, especialmente en la producción de cursos masivos en línea (MOOC) (Aguaded y Medina-Salguero, 2015; García-Roca y De Amo, 2021; Gertrudix y Rajas, 2016; Gordillo et al., 2019), donde se refina más el uso del vídeo como eje central del proceso didáctico. El uso de herramientas para la creación de contenido educativo para cursos en línea está dentro de los principios de la Edu-comunicación, que implica, por ejemplo, transformar lenguajes técnicos y/o disciplinares en narrativas que puedan llegar a todo público y tengan un valor didáctico. Es decir, que apoyen el cumplimiento de resultados de aprendizaje. Se describen, al menos dos enfoques relacionados, el primero es sobre enseñar sobre lectura crítica de medios de comunicación; otro desde la perspectiva de formar docentes y estudiantes para producir su propio contenido de apoyo a las clases. El material debe tener una intención educativa desde su diseño instruccional e involucra, en muchos casos, no solo a docentes, sino a equipos especializados de apoyo en la producción multimedial (Bermejo-Berros, 2021; Chiappe et al., 2016).

\section{MÉTODO}

El propósito de este estudio se centra en indagar en el funcionamiento de centros dedicados a la producción de vídeos educativos en instituciones educativas en Iberoamérica, así como el contexto sobre el uso de vídeo en educación.

Con base en el propósito de este estudio, se ha optado por un método cualitativo con investigación documental sobre el tema y el empleo de entrevistas semiestructuradas como un acercamiento a las experiencias de centros de producción de vídeos educativos, lo cual permita comprender el contexto y los procesos a través de la voz de los participantes (NIH, 2018).

\section{Participantes}

Para llegar a comprender cómo funcionan distintos centros para la producción de material educativo audiovisual se realizaron entrevistas en profundidad a miembros de los equipos de espacios de desarrollo de contenido audiovisual y medialabs ubicados en tres geografías diferentes: Colombia, México y España (Tabla 1) los cuales se seleccionaron con la técnica de muestreo aleatorio no probabilístico por conveniencia debido a la proximidad de los sujetos de estudio y porque presentan propuestas educativas que ponen el foco en la producción audiovisual y multimedia como protagonista de los procesos de innovación educativa. Las entrevistas se han 
realizado a directoras y/o personalidades con puestos de responsabilidad en cada uno de los centros.

\section{Tabla 1}

\section{Listado de participantes}

\begin{tabular}{llll}
\hline Participante & \multicolumn{1}{c}{ Centro } & \multicolumn{1}{c}{ Universidad } & País \\
Po1 & $\begin{array}{l}\text { Centro para la Excelencia Docente } \\
\text { (CEDU) }\end{array}$ & Universidad del Norte & Colombia \\
\hline P02 & VideoLab Tec & Tecnológico de Monterrey & México \\
\hline Po3 & MediaLab UHU & Universidad de Huelva & España \\
\hline
\end{tabular}

Fuente: elaboración propia.

\section{Instrumentos}

Los investigadores desarrollaron las preguntas de la entrevista, en la cual se han planteado diversas cuestiones relativas al origen del espacio de producción de materiales educativos digitales en cada una de estas universidades, a su método de trabajo, sus recursos tanto de equipamiento como humanos, además de incidir en los contenidos de mayor éxito y las carencias encontradas, con el objetivo de entender qué propuestas y mejoras serán necesarias para adaptarse a los nuevos escenarios educativos. De igual manera, se consultó por la construcción del concepto de innovación educativa a partir del uso de tecnologías de información y comunicación y cómo esto incide en el enfoque de la producción didáctica de los materiales.

\section{Figura 1}

Preguntas para la entrevista en profundidad

- ¿Qué servicio se ofrece?

- ¿Desde cuándo se ofrece?

- ¿Qué valor añade ese servicio a lo ya existente en la Universidad?

- ¿Por qué es necesario este servicio?

- ¿Cómo se desarrolla el trabajo?

- ¿Qué medios se emplean?

- ¿Qué contenido es el que más éxito tiene?

- ¿Qué contenido es el que menos éxito tiene?

- ¿Cuántas personas trabajan en crear estos contenidos?

- ¿Están satisfechos los usuarios con el servicio ofrecido?

- ¿Qué impacto tiene en la práctica en el aula y el desarrollo docente?

- ¿Cómo se puede mejorar?

- ¿Cómo se proyecta de cara al futuro?

- ¿Qué carencias se encuentran que deban ser solventadas?

Fuente: elaboración propia. 
Las entrevistas se realizaron entre noviembre y diciembre de 2020. Las tres entrevistas se realizaron por conferencia virtual (web-conference) con una duración promedio de 20 a 30 minutos. Un participante complementó las respuestas de forma escrita por correo electrónico. Los entrevistados fueron invitados a participar personalmente a través de una llamada telefónica y se les compartió el listado de preguntas.

Después, se han expuesto los datos obtenidos de las entrevistas aunando las respuestas a cada una de las preguntas, de manera que se pueda acceder a las similitudes y diferencias que se dan entre estos espacios. Finalmente, tras la observación de estos datos se ha realizado una discusión reflexionando acerca de lo encontrado.

En cuanto a las limitaciones del estudio, por tratarse de experiencias particulares, los resultados no pueden ser generalizados, sin embargo, dan un marco de referencia sobre lecciones aprendidas y retos sobre el tema. Esto supone que la discusión y conclusiones que se establezcan deben ser entendidas en relación con la muestra con la que se ha podido trabajar, siendo de interés, con proyección a futuro ampliar este estudio con más centros a fin de abordar en mayor profundidad la temática y de poder llegar a conclusiones más generales al respecto. Cada caso puede extrapolarse según distintos contextos y abarcar la problemática del video educativo desde distintos enfoques.

Asimismo, previamente, se ha realizado una revisión de literatura para tener mayor conocimiento del contexto de la evolución de la temática.

\section{RESULTADOS}

MediaLab, VideoLab, centros de innovación, laboratorios de apoyo... Si bien los nombres que se les atribuye a los espacios que se vienen describiendo no están bajo consenso, la finalidad de estos es similar. A continuación, vamos a exponer los resultados extraídos de las entrevistas a las personas con cargos de responsabilidad de los tres centros anteriormente mencionados.

\section{Origen}

Tanto en el caso de Colombia como en el de México, estos espacios tienen su origen en 2016. La Universidad del Norte cuenta con el Centro para la Excelencia Docente (CEDU) y una unidad de Diseño de Material Educativo Digital. Se producen materiales como vídeos, podcast, lecciones interactivas, aplicaciones, infografías, entre otros, que sirven de apoyo a la docencia. El antecedente de este servicio lo marca la unidad de nuevas tecnologías de la Universidad, que durante más de diez años asesoró a los docentes en el diseño y desarrollo de material digital. Por su parte, el Tec de Monterrey lleva treinta años brindando orientación y producción de recursos audiovisuales a la comunidad académica mediante la Dirección de 
Innovación Educativa. En 2013 inició el proceso de rediseño de su modelo educativo, llamado Modelo Educativo Tec21 (Figura 2), el cual ha detonado una transformación de sus integrantes y componentes. Desde la creación del VideoLab Tec, en 2016, ofrece de forma concreta: asesoría en el diseño y desarrollo de recursos educativos en vídeo para cursos de programas académicos, desarrollo de recursos en vídeo para alumnos y profesores y para responsables de áreas internas, e información sobre mejores prácticas en el uso de vídeo.

\section{Figura 2}

Componentes del Modelo Educativo Tec21

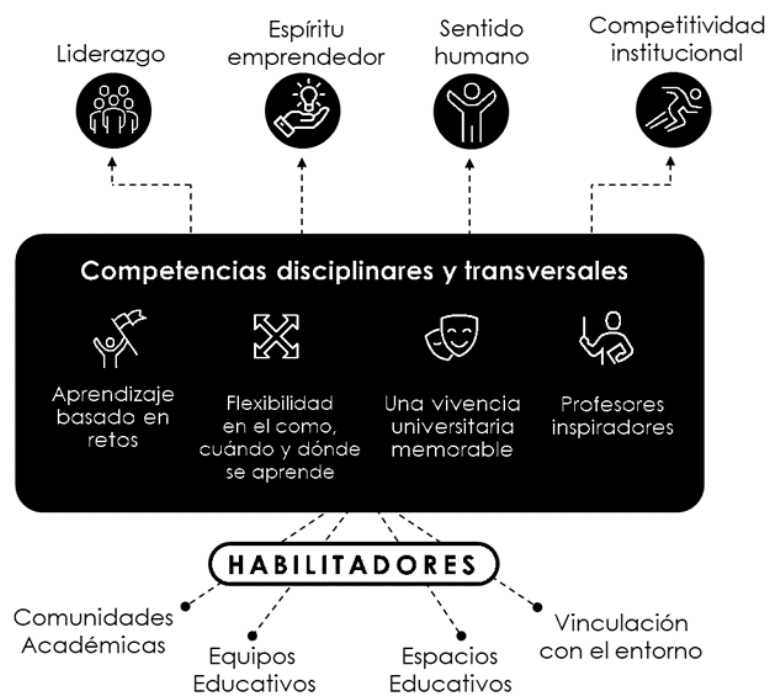

Fuente: Modelo Tec21. Tecnológico de Monterrey

MediaLab UHU, por otro lado, es un laboratorio más reciente. Comienza a forjarse en 2017, pero no es hasta finales de 2020 cuando se pone en marcha. Con anterioridad, no existía un servicio como el que ofrece este espacio en la Universidad de Huelva. Actualmente, el MediaLab se encuentra en una primera fase, enfocando su producción a los MOOC, trabajando de manera paralela en contenidos con diferentes targets. Este centro está destinado a la innovación y producción de contenidos audiovisuales y multimedia al servicio de la educación, de cara a la virtualización de la docencia y a la implementación de nuevos recursos que apoyen al profesorado y ofrezcan al alumnado nuevos métodos para acercarse al conocimiento. Se entiende este proyecto atendiendo al concepto de MediaLab que propone Salaverría (2015, p. 398), "unidades o departamentos dedicados a la investigación, experimentación, desarrollo e implementación de innovaciones tecnológicas y editoriales". Aunque 
al inicio estos laboratorios empezaran a desarrollarse vinculados, sobre todo, a empresas periodísticas, poco a poco las universidades han ido incorporando espacios de similares características con una perspectiva educativa e investigativa. Un ejemplo en el ámbito español es MediaLab UGR, solo que su rumbo se dirige más hacia el ámbito social que al audiovisual, aunque lo emplea también con iniciativas como Apps UGR y RadioLab UGR (Villa et al., 2020), y coincide en el carácter innovador, de experimentación y divulgación (Romero-Frías y Robinson-García, 2017).

\section{Valor añadido}

Este tipo de espacios ofrecen un valor añadido a los medios y servicios ya existentes en las universidades y a la vez suponen un impacto positivo en la actividad docente y en las experiencias en el aula. En estos tres laboratorios, se aboga por la creación de materiales educativos digitales para su posterior integración en el desarrollo de la docencia. El objetivo principal consiste en "ser una fuente de información y orientación para profesores, estudiantes y público en general, que desean producir sus propios vídeos educativos" (Tecnológico de Monterrey, 2021).

\section{Método de trabajo}

Por otro lado, hemos ahondado en los enfoques de trabajo de cada uno de estos centros. En este sentido, el CEDU se dirige principalmente al profesorado y lo acompaña en el proceso de desarrollo del material educativo, dividido en tres fases: diseño pedagógico, diseño comunicacional y desarrollo. El trabajo que se lleva a término en este espacio, se desarrolla de la siguiente manera: en la fase de asesoría pedagógica, se analizan las necesidades educativas frente a las cuales el profesor considera se debe desarrollar el material, se determina la estrategia pedagógica más apropiada para el uso del material dentro y fuera de la clase, se trabaja con el docente en la organización de las actividades de aprendizaje y contenidos, según las necesidades que exprese el docente con su asignatura. En la fase de diseño, se realizan los bocetos y maquetación del material. Se estructura la navegación, diseño gráfico, ubicación de módulos, fotografías y criterios comunicacionales como títulos, ubicación de módulos y herramientas complementarias. En la fase de desarrollo se genera el material educativo digital que se ha definido en las fases previas, con el equipo de ingenieros de sistema del CEDU, especializados en el uso educativo de las TIC.

MediaLab UHU está centrando, en este momento, su trabajo, en líneas generales, al desarrollo de diferentes MOOC, partiendo del siguiente procedimiento: primero, se establece la temática y se contacta con docentes e investigadores expertos en la materia; en segundo lugar, se pauta una estructura sobre la que se construirá el MOOC; tercero, se desarrolla el contenido literario y posteriormente el contenido 
audiovisual que constituirá tanto las unidades del curso como los materiales de apoyo del mismo. Finalmente, se lanza en la plataforma internacional MiriadaX.

En el caso del Tec, se consideran tres alcances con base en las necesidades que se han identificado de los usuarios, así como los requerimientos institucionales. En el primero, el sitio funge como una fuente de información con recursos abiertos para el autoaprendizaje de los usuarios, docentes o alumnos. La información está organizada en secciones de acuerdo con el proceso de producción de vídeo, preproducción, producción y post-producción. Asimismo, se incluye información sobre herramientas comerciales gratuitas o de pago de las cuales puede seleccionar según su interés y necesidad. En el segundo alcance se busca apoyar proyectos de profesores y estudiantes donde implementen el vídeo como recurso educativo; se les brinda asesoría para definir las características de su vídeo, identificar los recursos que requieren, y se comparten las mejores prácticas para que realicen un producto de calidad. En caso de que se solicite, también reciben acompañamiento en el desarrollo de su recurso. El tercer alcance está enfocado a fungir como un medio para compartir mejores prácticas y hacer una red de colaboración entre los usuarios. En el sitio se incluye la sección Experiencias Tec, en la cual los docentes comparten su experiencia con VideoLab Tec, de esta manera se busca acercar a los interesados en el uso de vídeo educativo para que intercambien experiencias entre pares.

\section{Recursos humanos y técnicos}

En cuanto a los medios y el personal que realizan las labores en estos estudios encontramos que el Tec de Monterrey cuenta con unas instalaciones para la realización de vídeo, ubicada en el Campus Monterrey. Esta sala cuenta con cabinas más pequeñas para la auto-grabación de vídeo, y pueden ser reservadas, así como se cuenta con el acompañamiento de una persona experta para asesorar en la producción del material. Además de este espacio, VideoLab Tec ofrece documentación sobre buenas prácticas en producción de vídeo mediante su web y asesoría online mediante e-mail, que ofrece la opción de una reunión virtual o presencial en el espacio. Estos medios están a disposición del personal docente e investigador a fin de fomentar la autoproducción de contenidos multimedia. No obstante, VideoLab Tec cuenta con un equipo base de cinco personas expertas en desarrollo de vídeo educativo y con un equipo extendido de trece personas, pertenecientes a la Dirección de Innovación Educativa, que desempeñan funciones de asesoría y producción de vídeo. Asimismo, de acuerdo con las características del proyecto que se trabaje, se pueden asignar más especialistas del equipo central.

El CEDU, tras años centrado en la producción de material, inició en 2017 una fase de cara a la oferta de servicio. Así, los docentes reciben la asesoría sobre planificación de clases, actividades y estrategias relacionadas con el uso de las TIC para enriquecer los ambientes de aprendizaje. Este espacio de la Universidad del Norte cuenta con un estudio de vídeo y audio que comparte con la Facultad de Diseño 
Gráfico. Todos los materiales quedan a disposición de los docentes y estudiantes a través del LMS (Blackboard). En el caso de los vídeos, también quedan abiertos en el canal del CEDU en YouTube, donde tienen alta demanda de visitas, contando con más de 16.000 suscriptores a diciembre de 2020. Este servicio cuenta con un grupo base de seis personas, pudiendo llegar a equipos de diez personas para la realización de proyectos: comunicadores, docentes, diseñadores pedagógicos, diseñadores gráficos, productores de vídeo, especialistas en multimedia. Además, cuenta con puestos para prácticas universitarias, fomentando así el aprendizaje de los propios estudiantes de Universidad del Norte de carreras como Comunicación Social con énfasis en producción audiovisual y Diseño Gráfico.

El MediaLab UHU dispone de un estudio de grabación, que contiene un ciclorama de color verde para la realización de chromas y la última tecnología para la realización, producción y edición de vídeo y audio. Además, se ofrece a los interesados en la realización de MOOC asesoría sobre la estructuración y creación de contenidos educativos. Este laboratorio de medios se encuentra ubicado en la Facultad de Educación, y es coordinado por los principales investigadores del Grupo de Investigación Ágora, especializados en edu-comunicación, lo cual aporta una base científica al desarrollo y la puesta en práctica de los cursos realizados. Se cuenta, además, con dos expertos especializados en la producción de contenidos audiovisuales, responsables de las grabaciones, edición y montaje de los vídeos, así como alumnos en prácticas a los que se forma en el desempeño de estas labores.

\section{Satisfacción, éxito y alcance}

En lo que respecta a la satisfacción de los usuarios con los servicios ofrecidos, el CEDU cada año realiza a los docentes una encuesta de satisfacción del servicio, alcanzando una puntuación de 4,8 sobre 5 , lo que indica la valoración positiva de los usuarios: "La estrategia de acompañamiento está muy bien organizada. Se tiene un equipo muy capacitado, se generan los espacios de revisión y asesorías adecuados y se realizan retroalimentaciones pertinentes" (Docente de Ing. civil). El éxito del material que se produce se mide de distintas maneras. Por una parte, se observan las consultas a los materiales, teniendo los vídeos educativos un gran alcance. Los vídeos tutoriales creados en áreas como Ciencias Básicas y Áreas Médicas suelen ser los más consultados. Por otra parte, se contabilizan los suscriptores en el canal de YouTube y las visitas de los vídeos. También se hace acompañamiento al uso de material en clase, realizando encuestas y grupos focales con estudiantes para conocer su percepción sobre el cumplimiento de los resultados de aprendizaje.

El VideoLab Tec actualmente no cuenta con una encuesta de satisfacción para medir la opinión de los usuarios; los profesores que han accedido a publicar un testimonial de su experiencia en el sitio expresan estar satisfechos con la asesoría y acompañamiento recibido. En cuanto al éxito y el alcance, durante el 2020 se tuvieron en la web alrededor de 1.000 visitantes mensuales. En la siguiente tabla 
(Tabla 2) se enumeran las páginas más visitadas durante el 2020 que se refieren, principalmente, a procedimientos, herramientas y tutoriales para la producción de vídeos.

\section{Tabla 2}

Reporte de usuarios de VideoLab Tec durante 2020

\begin{tabular}{|c|c|c|c|c|}
\hline Visitantes & $\begin{array}{l}\text { Páginas } \\
\text { vistas }\end{array}$ & Top de páginas visitadas & $\begin{array}{c}\text { Países } \\
\text { visitantes }\end{array}$ & $\begin{array}{c}\text { Top } 10 \text { países } \\
\text { visitantes }\end{array}$ \\
\hline 11.466 & 58.985 & $\begin{array}{l}\text { 1. ¡Haz tus propios vídeos! } \\
\text { 2. Guía paso a paso } \\
\text { 3. Acerca del vídeo educativo } \\
\text { 4. Herramientas y tutoriales } \\
\text { 5. Herramientas para grabar y editar } \\
\text { vídeos } \\
\text { 6. Acerca de } \\
\text { 7. Moovly: crea vídeos en línea } \\
\text { 8. Canales de profesores } \\
\text { 9. Para crear presentaciones y vídeos } \\
\text { animados } \\
\text { 10. Antes de grabar tu vídeo (pre- } \\
\text { producción) }\end{array}$ & 40 & $\begin{array}{l}\text { 1. México } \\
\text { 2. EE. UU. } \\
\text { 3. } \text { Colombia } \\
\text { 4. } \text { Chile } \\
\text { 5. Perú } \\
\text { 6. Francia } \\
\text { 7. Alemania } \\
\text { 8. Irlanda } \\
\text { 9. Argentina } \\
\text { 10. España }\end{array}$ \\
\hline
\end{tabular}

Fuente: VideoLab Tec. Dirección de Innovación Educativa. 2020.

Por el contrario, el contenido de menor éxito son los formatos y la producción de eventos. Los formatos descargables incluyen cartas legales para la cesión de derechos, cortinillas, plantillas para redactar un guion de vídeo, información sobre los lineamientos de identidad institucional, plantillas para incluir pantallas de créditos y plantillas en PowerPoint. La producción de eventos es un servicio que se ofrecía a través de la Dirección de Innovación Educativa, sin embargo, el enfoque se ha ajustado a eventos de estrategias institucionales por lo que la solicitud se recibe a través de áreas directivas y no de peticiones individuales.

Es preciso señalar que, debido al estado de fase inicial de MediaLab UHU, aún no se puede obtener una respuesta acerca de la satisfacción, el éxito, el alcance o sobre los aspectos que pueden ser susceptibles de mejora, del mismo modo que la detección de carencias.

\section{Retos a superar}

Ante las mejoras y las carencias encontradas, en VideoLab Tec encuentran necesaria la actualización constante de nuevos contenidos relacionados con la inclusión de tendencias en el campo de la producción y en el diseño de recursos didácticos. Así como en el propio diseño de los contenidos del sitio que sirvan como 
referencia de recursos bien diseñados, atractivos visualmente y con una intención bien logradas. Además, es oportuno aumentar la documentación sobre buenas prácticas en el uso de vídeo, herramientas y tutoriales para continuar siendo relevantes para los usuarios. Así como la inversión de tiempo en el diseño y la actualización y, sobre todo, la definición de estrategias que apoyen la difusión y participación de más docentes.

ElCEDU determina imprescindible tener en cuenta que el tiempo en la elaboración del material puede extenderse si no se cuenta con los diseños pedagógicos a tiempo. Esto depende mucho de los cronogramas de los docentes que priorizan su actividad en relación a la docencia y la investigación, por ello, es conveniente revisar planes de incentivos para que participen del programa con más consistencia. De igual manera se prevé contar con más diseñadores pedagógicos que apoyen el proceso, especialmente pensando en producir a futuro materiales para el diseño de ambientes virtuales en aumento.

\section{Proyección a futuro}

Por último, de cara a la proyección futura, el CEDU entra a apoyar -de cara a la pandemia - el proceso de creación de aulas virtuales en pre-grado y posgrado. Esto implica el apoyo para el diseño del modelo de virtualización de la universidad, la formación de docentes y la proyección de la modalidad en la institución. También la articulación de todas las instancias curriculares y administrativas para las adaptaciones. Teniendo en cuenta que es una universidad presencial, se requieren ajustes en distintas unidades. ElVideoLab Tec proyecta su laboratorio como un espacio con más contenidos de valor, pero, sobre todo, con un impacto mayor y más evidente en transformar esta práctica de los docentes y que, consecuentemente, se refleje en el consumo de estos materiales contenidos y que sea medible a través de indicadores como visualizaciones, descargas, número de profesores interesados en participar, entre otros. Además, se tratará de crear alianzas con áreas internas o externas a la institución para un mejor aprovechamiento. El MediaLab UHU entiende que el futuro de la educación se plantea como un híbrido entre las modalidades presenciales y virtuales, por lo que será preciso enfocar el estudio hacia la puesta en marcha de mayor contenido educativo de calidad con un enfoque dinámico y actualizado que incida en las necesidades tanto de alumnos como de docentes e investigadores en vistas a una mejora en la experiencia educativa mediante la adaptación multimedia, audiovisual y digital.

\section{DISCUSIÓN}

Los centros de producción de materiales educativos digitales surgieron en las instituciones educativas como un espacio en el que docentes y estudiantes disponen de equipo especializado para el desarrollo de recursos de vídeo. En parte se debe a la 
relevancia que ha tenido el uso de vídeo en la última década con el surgimiento de los MOOC (cursos masivos abiertos en línea), así como de plataformas que facilitaron su publicación como YouTube y Facebook, además del uso de la telefonía móvil y acceso a la banda ancha (Choe, 2017; Villa et al., 2020). Tal es el caso de los centros revisados en esta investigación, que en esta última década han dedicado recursos humanos y tecnológicos con el fin de contribuir a la formación de la comunidad académica en el desarrollo de recursos de vídeo de calidad, y como resultado tanto docentes como estudiantes se han vuelto productores y consumidores de recursos digitales (Villa et al., 2020). La evolución de la tecnología ofrece nuevas posibilidades y aplicaciones educativas para los usuarios.

La producción de vídeo se ha caracterizado por requerir equipamiento y espacios especializados, además de personal entrenado. Sin embargo, en los últimos años se han desarrollado softwares y equipos que resultan más intuitivos para el usuario principiante (Goodwin y Lim, 2016; Woolfitt, 2015), por lo que puede producir recursos de calidad. En este estudio las instituciones educativas coinciden en la importancia de definir las necesidades educativas a cubrir con los materiales educativos, ya sea que esta orientación se brinde a través de los expertos del centro, o con materiales para el autoaprendizaje disponibles en línea, lo cual es una alternativa que brinda flexibilidad para que los docentes y alumnos aprendan a su ritmo (Hajhashemi et al., 2018).

No obstante, cada vez más, la tendencia apunta a centros o medialabs enfocados en el apoyo al profesor para que realice curaduría de contenido existente o elabore sus propios materiales con software gratuito, inclusive con su celular y desde cualquier dispositivo personal. Aunque existen recursos educativos disponibles en internet, por ejemplo, el canal del CEDU en YouTube cuenta con colecciones de vídeo de diversas disciplinas, el profesor requiere invertir tiempo en la curaduría para encontrar los recursos que se adapten mejor al perfil de sus estudiantes y necesidades de aprendizaje, lo que ha detonado el interés de algunos docentes por desarrollar sus propios recursos de vídeo (Rengel et al., 2019). Esto fue evidente con la Pandemia del COVID-19, ya que fue inminente el traslado de la enseñanza a espacios de aprendizaje virtuales y en línea y muchos profesores, ante la imposibilidad de ir al campus o contar con apoyo especializado, prepararon sus propios materiales, como ejemplo, en el sitio de VideoLab Tec se registraron en promedio 1.00o visitantes promedio mensuales durante el 2020. Las herramientas de autoproducción de material promueven la autonomía, haciendo que el profesor dependa cada vez menos de los centros de apoyo.

Otro aspecto relevante observado, es que, gradualmente, los centros construyen con mayor frecuencia materiales educativos interactivos, que promueven la participación del público, generan comunidad y activan el principio de aprendizaje colaborativo en red como es el caso de MediaLab UHU donde, además de investigadores, participan alumnos en prácticas para el desarrollo de recursos de vídeo, lo cual les permite tener experiencias en equipos interdisciplinarios donde 
desarrollan habilidades de utilidad para su futura carrera profesional (Villa et al., 2020).

Un reto tanto para docentes como para los centros o medialabs será mantenerse actualizados en el diseño y desarrollo de recursos de vídeo, aprovechando los avances tecnológicos, así como pedagógicos. La producción de vídeo de calidad requiere atender las características propias de este formato, a la vez que a la intención educativa para la cual se desarrolla el vídeo. La satisfacción que los usuarios perciban con los servicios ofrecidos y del material disponible son aspectos que los tres centros consideran como parte de su operación y para la mejora continua de sus actividades.

Se puede prever que los centros o medialabs, a futuro, podrían orientar sus servicios al acompañamiento en el diseño pedagógico de los materiales y no tanto en su producción técnica, brindando herramientas y soporte para la construcción de secuencias didácticas y en el diseño de experiencias de aprendizaje de calidad virtuales y/o presenciales. Se necesita también más investigación acerca de cómo el vídeo puede apoyar el diseño de ambientes virtuales desde el enfoque del aprendizaje activo y constructivista.

Los resultados aquí presentados pueden ser de utilidad para otras universidades y centros interesados en incursionar en la producción de vídeo, así como para encontrar oportunidades de colaboración que coadyuven a enriquecer la experiencia de aprendizaje y la formación docente.

\section{CONCLUSIONES}

La adopción de herramientas tecnológicas por parte de docentes y estudiantes detonó el uso del video educativo en el proceso de enseñanza-aprendizaje, y ante esto, algunas instituciones educativas han desarrollado estrategias para asegurar la calidad de los recursos. El propósito de esta investigación fue indagar el funcionamiento de centros dedicados a la producción de video educativo en instituciones educativas en Iberoamérica y se seleccionaron tres centros de esta región por su enfoque en la producción audiovisual.

Con base en los resultados obtenidos en las entrevistas realizadas, podemos concluir que los tres centros cuentan con el servicio de producción de material audiovisual educativo; CEDU desde su unidad de Diseño de Materiales y VideoLab Tec centran sus esfuerzos, en mayor medida, en el asesoramiento y acompañamiento a docentes e investigadores que quieran realizar videos educativos y otro tipo de material digital. Por su parte, MediaLab UHU se encara más a la producción audiovisual educativa, haciéndose cargo desde este espacio del trabajo de producción y realización, y asesorando en el caso de la redacción de guiones. Esto resulta de interés para otras instituciones educativas en cuanto a que deben definir el alcance y servicios que ofrecen para dar claridad a sus usuarios, así como a la institución.

Asimismo, tener claridad en los servicios conlleva a la planificación del recurso humano y técnico requerido para su implementación. Se observan mayores 
similitudes entre los dos centros iberoamericanos, que a su vez son los más longevos tanto en el perfil del recurso humano, así como en los recursos técnicos como plataformas de publicación de video. Siendo notable el escaso rodaje del centro de la Universidad de Huelva, que aún se encuentra sumergido en el primer proyecto y, por lo tanto, parte de un enfoque inicial que no puede saberse el devenir que tomará. Es importante destacar que CEDU y MediaLab UHU incluyen la participación de estudiantes en prácticas, lo cual puede resultar una alternativa plausible para otras universidades; además, dado que la producción de video va de la mano de recursos técnicos, las instituciones educativas deben considerar el desarrollo de un caso de negocio/estudio de factibilidad (business case) para asegurar contar con los recursos financieros a través del tiempo y así tener el recurso humano requerido y brindar el mantenimiento y actualización técnicos necesarios de los centros.

La satisfacción del servicio percibido por los usuarios, así como la mejora continua, basada en evidencia es una estrategia que ayuda a enfocar los recursos donde se requiere. CEDU cuenta con instrumentos cualitativos (grupos de enfoque) y cuantitativos (encuestas) que aplica sistemáticamente, mientras que VideoLab Tec utiliza una práctica cualitativa (testimoniales) optativa para sus usuarios, y para el seguimiento al uso de recursos, contabiliza visitas y descargas al igual que CEDU. MediaLab UHU aún no cuenta con resultados de satisfacción; sin embargo, las prácticas de los centros pueden servir como una guía para plantear lo que funcione para sus servicios.

Cabe señalar que los tres centros estudiados plantean enfoques innovadores de cara a la virtualización y mediación de la educación y el conocimiento, siendo esto un reclamo y una necesidad hallada en el ámbito educativo, especialmente tras la situación acontecida durante la pandemia del COVID-19, que ha permitido vislumbrar el camino que queda por recorrer en este sentido. Este estudio brinda una perspectiva de los centros dedicados a la producción de video educativo en instituciones educativas en Iberoamérica y, más que una propuesta del "deber ser", se plantean elementos importantes a considerar por tomadores de decisiones, investigadores, así como docentes interesados en el desarrollo de estos centros para que los adecuen a su realidad y necesidades.

\section{REFERENCIAS}

Accino, J., y Cebrián, M. (2008). La plataforma es la red: Aprendizaje centrado en el usuario y arquitecturas centradas en la identidad. Enfoques, 84, 37-47. https:// bit.ly/2IEAyKP

Aguaded, I., y Medina-Salguero, R. (2015). Criterios de calidad para la valoración y gestión de MOOC. Revista Iberoamericana de Educación a
Distancia, 18(2), 119-143. https://doi. org/10.5944/ried.18.2.13579

Anderson, D. R., y Davison, M. C. (2019). Receptive versus interactive video screens: A role for the brain's default mode network in learning from media. Computers in Human Behavior, 168-180. https://doi. org/10.1016/j.chb.2019.05.008 
Anderson, T., y Dron, J. (2011). Three generations of distance education pedagogy. The International Review of Research in Open and Distributed Learning, 12(3), 80-97. https://doi. org/10.19173/irrodl.v12i3.890

Aparici, R. (1997). La revolución de los medios audiovisuales. Editorial de la Torre.

Bermejo-Berros, J. (2021). The critical dialogical method in educommunication to develop narrative thinking. Comunicar, 29(67). https://doi.org/10.3916/C672021-09

Bravo-Ramos, L. (1996). ¿Qué es el vídeo educativo? Comunicar, 6, 100-105. https://doi.org/10.3916/Co6-1996-20

Buckingham, D. (2003). Media Education, learning and Contemporary Culture. Polity Press.

Carmichael, M., Reid, A., y Karpicke, J. (2018). Assessing the impact of educational video on student engagement, critical thinking and learning: The current state of play. SAGE Publishing. https://bit.ly/39q3Lt5

Cebrián, M. (2002). Los vídeos didácticos: claves para su producción y evaluación. https://bit.ly/2Ds8j2S

Cebrián, M. (2009). Nuevas formas de comunicación: Cibermedios y medios móviles. Comunicar, 17(33), 10-13. https://doi.org/10.3916/c33-2009-01$\underline{001}$

Chiappe, A., Rozo, H., Menjivar, E., Corchuelo, M., y Alarcón, M. (2016). Edu-comunicación en entornos digitales: una mirada desde la comunicación. En, C. Parra (Ed.), Doctorado en Educación: temas y conceptos. (pp. 159-177). Chía: Universidad de la Sabana. https://bit. ly/2QW2VKo

Choe, E. (2017). Optimizing video for Learning: A case study-based primer of informal, educational, digital video best practices. Massachusetts Institute of Technology. https://doi.org/10.2139/ ssrn.2909769
Daza, G. (2012). Historia y perspectivas del vídeo educativo y cultural en América Latina. Diálogos.

Edu Trends (Ed.) (2017). Storytelling. Monterrey: Observatorio de Innovación Educativa del Tecnológico de Monterrey. https://bit.ly/2JkqAmg

Fedorov, A. (2010). Media education practices in teacher training. Acta Didáctica Napocensia, 3(3), 57-70. https://ssrn.com/abstract=2624237

Ferrés, J. (1992). Vídeo y educación. Paidós.

Gabelas, J. (2007). Una perspectiva de la educación en medios para la comunicación en España. Comunicar, 15(28), 69-73. https://www.revistacomunicar.com/ojs/ index.php/comunicar/article/view/C282007-09

García, M. (2014). Uso instruccional del vídeo didáctico. Revista de Investigación, 38(81), 43-68. https://bit.ly/3ujFvjX

García-Roca, A., y De-Amo, J. M. (2021). Juvenile literary hypertextual fanfiction: Evolution, analysis and educational possibilities. Psychology, Society and Education, 11(2), 241-251. https://doi. org/10.25115/psye.v11i2.2187

Gertrudix, M., y Rajas, M. (2016). Narrativa audiovisual: Producción de vídeos colaborativos para MOOC. Opción, 32(12), 349-374. https://bit.ly/2tTDTQG

Goodwin, M. y Lim, L. (2016). MediaLab Report Melbourne: School of culture \& communication. The University of Melbourne. https://bit.ly/3cJqsdV

Gordillo, A., López-Pernas, S., y Barra, E. (2019). Effectiveness of MOOCs for teachers in safe ICT use training. [Efectividad de los MOOC para docentes en el uso seguro de las TIC]. Comunicar, 61, 103-112. https://doi.org/10.3916/C612019-09

Guerrero-Romera, C., Sánchez-Ibáñez, R., Escribano-Miralles, A., y Vivas-Moreno, V. V. (2021). Active teachers' perceptions on the most suitable resources for teaching history. Humanities and Social 
Sciences Communications, 8(1). https:// doi.org/10.1057/s41599-021-00736-7

Hajhashemi, K., Caltabiano, N.J.,y Anderson, N. (2018). Lecturers' perceptions and experience of integrating online videos in higher education. Australian Educational Computing, 33(1). https://bit.ly/3rKjWIc

Hovious, A., Shinas, V. H., y Harper, I. (2021). The compelling nature of transmedia storytelling: Empowering twenty firstcentury readers and writers through multimodality. Technology, Knowledge and Learning, 26(1), 215-229. https:// doi.org/10.1007/s10758-020-09437-7

Hung, I. C., Kinshuk, y Chen, N. S. (2018). Embodied interactive video lectures for improving learning comprehension and retention. Computers \& Education, 117, 116-131. https://doi.org/10.1016/j. compedu.2017.10.005

Koumi, J. (2006). Designing video and multimedia for open and flexible learning. Routledge. https://doi. org/10.4324/9780203966280

Laaser, W., y Toloza, E. (2017). The changing role of the educational video in higher distance education. The International Review of Research in Open and Distributed Learning, 18(2), 264276. https://doi.org/10.19173/irrodl. v18i2.3067

NIH Office of Behavioral and Social Sciences Research. (2018). Best practices for mixed methods research in the health sciences (2nd ed). National Institutes of Health. https://obssr.od.nih.gov/sites/ obssr/files/Best Practices for Mixed Methods Research.pdf

Rengel, R., Pascual, E., Íñiguez-de-la-Torre, I., Martín, M. J., y Vasallo, B. G. (2019).
Experiences on the design, creation, and analysis of multimedia content to promote active learning. $J$ Sci Educ Technol, 28, 445-451. https://doi.org/10.1007/ s10956-019-09777-9

Romero-Frías, E., y Robinson-García, N. (2017). Laboratorios sociales en Universidades: Innovación e impacto en Medialab UGR. Comunicar, 51, 29-38. https://doi.org/10.3916/C51-2017-03

Salaverría, (2015). Los labs como fórmula de innovación en los medios. Profesional de la Información, 24(4), 397-404. https:// doi.org/10.3145/epi.2015.jul.06

Salinas-Ibáñez, J., y De-Benito, B. (2020). Construction of personalized learning pathways through mixed methods. [Construcción de itinerarios personalizados de aprendizaje mediante métodos mixtos]. Comunicar, 65, 31-42. https://doi.org/10.3916/C65-2020-03

Scavarelli, A., Arya, A., y Teather, R. J. (2021). Virtual reality and augmented reality in social learning spaces: A literature review. Virtual Reality, 25(1), 257-277. https:// doi.org/10.1007/s10055-020-00444-8

Tecnológico de Monterrey (Ed.) (11-022021). Acerca de nosotros. VideoLab Tec. https://innovacioneducativa.tec.mx/es

Villa, M. I., Marulanda, A., y Molina, T. (2020). La experimentación educativa, social y técnica en los medialabs universitarios. Revista Complutense de Educación, 31(2), 231-240. https://doi. org $/ 10.5209 /$ rced.62114

Woolfitt, Z. (2015). The effective use of video in higher education. Nederland: Lectoraat Teaching, Learning and Technology. Inholland University of Applied Sciences. https://bit.ly/3fkgC6I

\section{PERFIL ACADÉMICO Y PROFESIONAL DE LOS AUTORES}

María Toscano-Alonso. Diseño y estructuración de MOOC, creación audiovisual, narrativa audiovisual, estudios de género. https://orcid.org/oooo0002-7263-3400

E-mail: maria.toscano.alonso@gmail.com 
Ignacio Aguaded Gómez. Media literacy, competencia mediática, tecnologías educativas, ICT, TIC. https://orcid.org/0000-0002-0229-1118

E-mail: aguaded@uhu.com

\section{DIRECCIÓN DE LOS AUTORES}

Facultad de Ciencias de la Educación, Psicología y Ciencias del Deporte Universidad de Huelva Avda. Tres de Marzo, s/n, 21007 Huelva (España)

Edna M. Manotas-Salcedo. Diseño de materiales digitales, educación virtual, innovación pedagógica, formación docente en educación superior. https://orcid. org/0000-0002-4080-2740

E-mail: ednam@uninorte.edu.co

DIRECCIÓN DE LA AUTORA

Universidad del Norte

Km5. Vía Puerto Colombia.

Barranquilla

Silvia C. Farias-Gaytán. Innovación educativa, educación virtual, gestión de proyectos educativos. https://orcid.org/0000-0001-5858-5900

E-mail: silvia.farias@tec.mx

DIRECCIÓN DE LA AUTORA

Tecnológico de Monterrey

Ave. Eugenio Garza Sada No. 2501 Sur Col. Tecnológico.

Monterrey. C.P. 64849.

Nuevo León (México)

Fecha de recepción del artículo: 07/04/2021

Fecha de aceptación del artículo: 06/07/2021

Fecha de aprobación para maquetación: 28/09/2021 